\title{
RANDOM EQUIVALENT INITIAL BOW AND TILT IN STEEL FRAME
}

\author{
A. Machowski ${ }^{1}$ and I. Tylek ${ }^{2, *}$ \\ ${ }^{1}$ Assoc. Prof. D. Sc. Ph. D., Faculty of Civil Engineering, \\ Cracow University of Technology, ul. Warszawska 24, 31-155 Cracow, Poland, \\ ${ }^{2}$ Assist. Prof. Ph. D., Faculty of Civil Engineering, \\ Cracow University of Technology, ul. Warszawska 24, 31-155 Cracow, Poland \\ *(Corresponding author: E-mail: ik2@poczta.fm)
}

Received: 3 September 2011; Revised: 26 September 2011; Accepted: 30 September 2011

\begin{abstract}
Probabilistic models of equivalent geometrical imperfections suitable to analysis of steel buildings frames are under consideration. Statistically based models of equivalent imperfections, besides of application in structural reliability analysis, should be taken as basis of verification of equivalent imperfection formulas and rules of introducing imperfections into frame calculations proposed in different Standards. Column random equivalent initial bow was obtained in this paper utilizing randomization of buckling coefficient in well-known deterministic formula of equivalent initial bow. Problem of random equivalent initial tilt of frame was considered for spatial mechanical model of skeletal structure, braced by rigid floor disks, using results of existing buildings geodesic measurements statistical analysis. In the paper assessment of random initial tilts and initial bows joint effect is commented.
\end{abstract}

Keywords: Multistory steel frame, Random equivalent geometrical imperfection, Eurocode 3

\section{INTRODUCTION}

Carrying capacity of steel frames significantly depends on imperfections (Table 1(a)): single column; residual stresses and axes misalignments as well as bar system imperfections; column out-of-plumbs, eccentricity in column field joints, assembly stresses, random joint rigidity.

In traditional approach (Chen and Toma [1], Narayanan [2]) imperfections are modeled by linear combination of buckling modes (simplifying - only dominant buckling mode) which correspond to solution of bifurcation problem using linearized model of perfect system elastic stability. In this approach real structure imperfections are taking into consideration only indirectly - by using standard buckling curves. Column out-of-plumbs and other important imperfections, arising during assembly, are passed over in traditional approach.

Contemporary standard approach depends on taking into account of two equivalent geometrical imperfections (Table 1(b)): equivalent initial column bow $e_{0}$ (column misalignment and column residual stresses) and equivalent initial frame tilt $\phi_{0}$ (column out-of-plumbs, eccentricity in column field joints, assembly stresses, randomness of beam-column joints rigidity).

Standard approach approximately takes into consideration steel skeleton fabrication conditions, in particular (Figure 1): columns division on assembly parts and its assembly with random out-of-plumbs - causing significant changes of moment values in equilibrium equations - because of large vertical forces occurrence. 
Table 1. Conceptions of Equivalent Geometric Imperfections

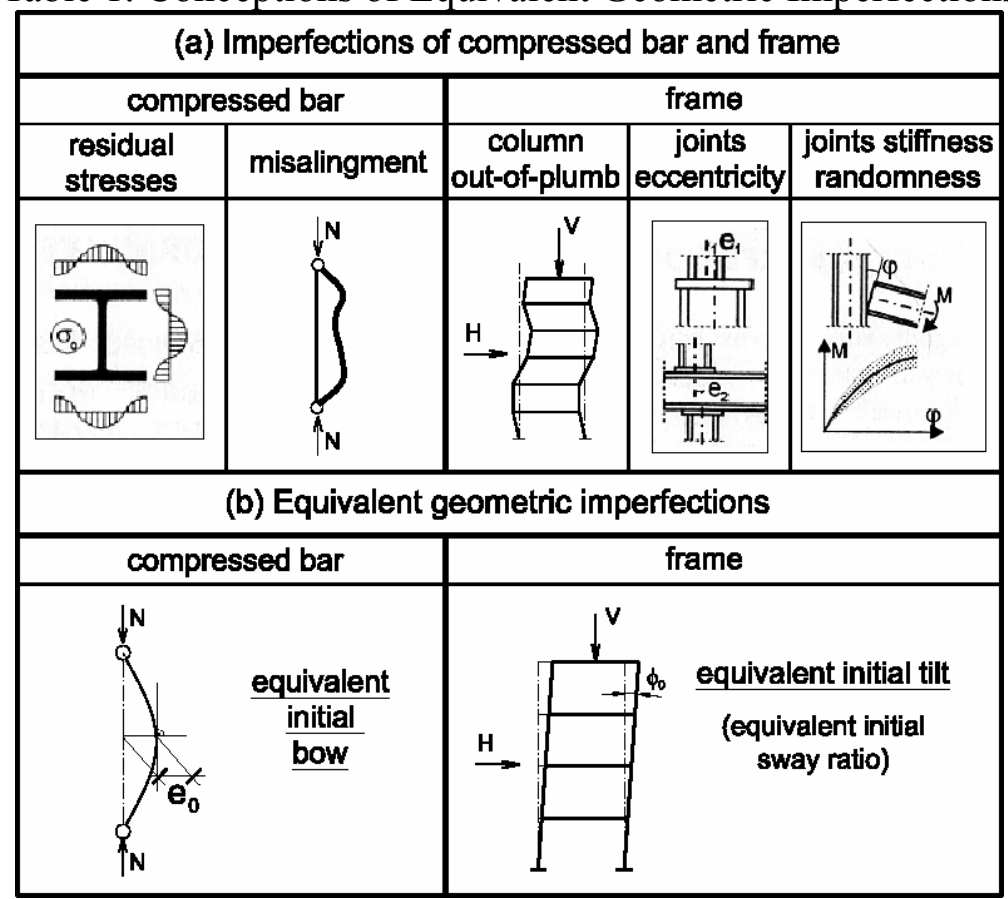

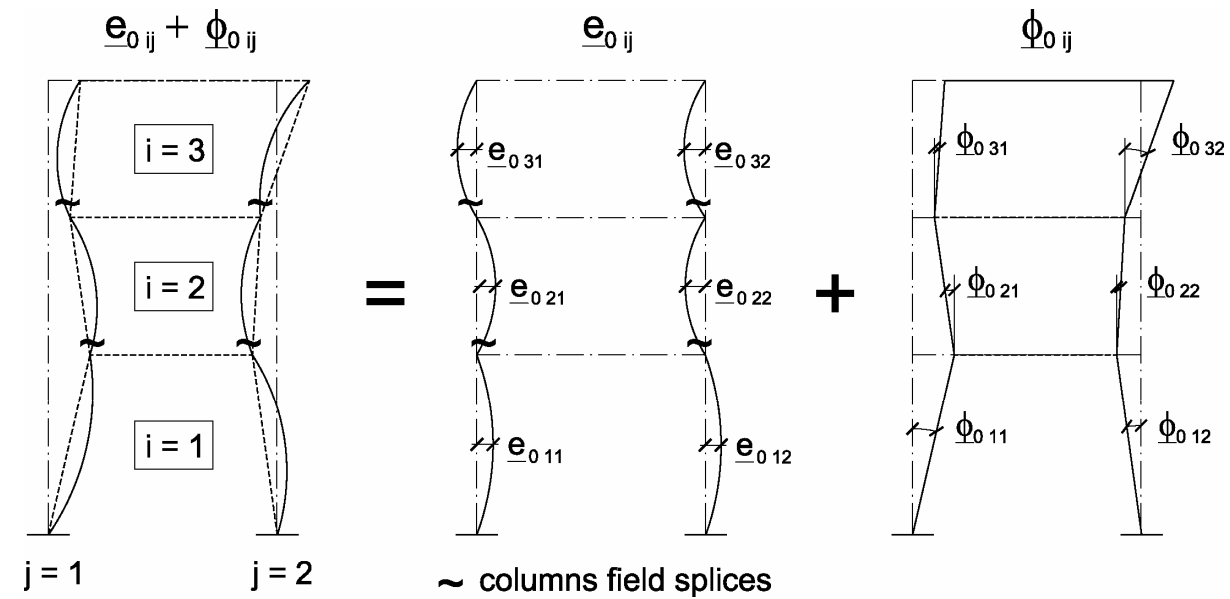

Figure 1. Columns Random Initial Bows and Initial Tilts

Issues connected with research and modeling of equivalent geometrical imperfections $e_{0}$ and $\phi_{0}$ are discussed in chapters 2 to 4 of this paper.

\section{RANDOM EQUIVALENT INITIAL BOW OF COLUMN}

Random equivalent initial bow of column is obtained as function of random buckling coefficient $\underline{\varphi}$ (random values are underlined).

Random buckling coefficient was determined in papers (Machowski, A. [3]) utilizing Fukumoto and Itoh statistical data (Fukumoto, Y. and Itoh, Y. [4]) and applying randomization of axially compressed steel bar flexural buckling resistance standard formula

$\underline{N}_{b . R}=\underline{\varphi} \cdot \underline{N}_{p l}$. 
It was assumed that random flexural buckling resistance of compressed steel bar $\underline{N}_{b . R}$ and bar cross-section random plastic resistance $\underline{N}_{p l}$ have logarithmic-normal distribution ${ }^{(*)}-$ stable (invariant) with respect to multiplication and defined only for positive values of $\underline{N}_{b . R}$ and $\underline{N}_{p l}$.

It follows that stochastically independent (originally) from $\underline{N}_{p l}$ buckling coefficient $\underline{\varphi}$ has also logarithmic-normal distribution. Flexural buckling resistance $\underline{N}_{b . R}$ has median equal to product of medians $\breve{N}_{b . R}=\breve{\varphi} \cdot \breve{N}_{p l}$ and logarithmic coefficient of variation equal to vector sum of logarithmic coefficients of variation $v_{N b . R}=\sqrt{v_{\varphi}^{2}+v_{N p l}^{2}}$.

Appropriate sets of data have been statistically processed with a mean-square approximation of experimental values of medians $\breve{\varphi}(\Lambda)$ and logarithmic coefficients of variation $\breve{v}_{\varphi}(\Lambda)$, where $\Lambda=\sqrt{\breve{N}_{p l} / \breve{N}_{E}}$ - relative slenderness $\left(\breve{N}_{E}\right.$ - median of bar Euler's resistance) and application of formulas:

$$
\breve{\varphi}(\Lambda)=\left(1+\Lambda^{2 n}\right)^{-\frac{1}{n}}, \quad \breve{v}_{\varphi}(\Lambda)=v_{m} \cdot\left(\frac{2 \cdot l \cdot \Lambda}{1+(l \cdot \Lambda)^{2}}\right)^{2} .
$$

Empirical parameters (Machowski, A. [3]): n, $v_{m}$, and $l$ for buckling curves: "a", "b" and "c" (CEN [5], PKNMiJ [6]) are presented in Table 2.

Table 2. Empirical parameters for formula (2)

\begin{tabular}{|l|l|l|l|}
\cline { 2 - 4 } \multicolumn{1}{c|}{} & \multicolumn{3}{|c|}{ Buckling curve } \\
\cline { 2 - 4 } \multicolumn{1}{c|}{} & $\mathrm{a}$ & $\mathrm{b}$ & $\mathrm{c}$ \\
\hline$n$ & 3,0 & 2,6 & 2,2 \\
\hline$v_{m}$ & 0,10 & 0,11 & 0,13 \\
\hline$l$ & 0,8 & 0,9 & 1,0 \\
\hline
\end{tabular}

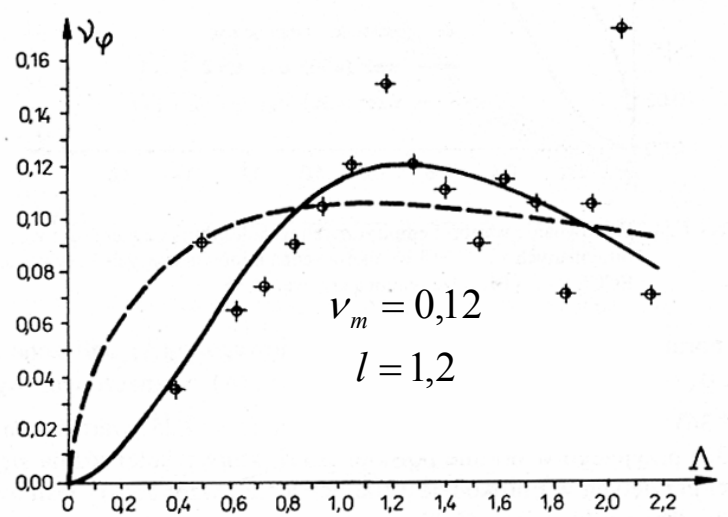

Figure 2. The Example of Values $v_{\varphi}(\Lambda)$ Approximation for $N=1251$

Column Research Council Test Results (Machowski [3])

$\left(^{*}\right)$ parameters of logarithmic-normal variable $\underline{X}>0$ with probability density function $f(x)=\frac{1}{\sqrt{2 \pi} \cdot v_{X} \cdot X} \cdot \exp \left(-\ln ^{2}(X / \bar{X}) /\left(2 \cdot v_{X}^{2}\right)\right) ;$ mean value $\bar{X}=E\{X\}$ and coefficient of variation $\mathrm{v}_{X}$ are related as follows: $\breve{X}=\bar{X} / \sqrt{1+\mathrm{v}_{X}^{2}}, v_{X}=\sqrt{\ln \left(1+\mathrm{v}_{\mathrm{X}}^{2}\right)}$. 
An example of experimental coefficients of variation approximation by means of function $v_{\varphi}(\Lambda)$ according to Eq. 2 is shown in Figure 2; single points represent empirical mean values of $v_{\varphi}^{*}(\Lambda)$ connected with individual slenderness $\Lambda$. Form of function (2) refers to earlier proposals connected with stability coefficients (PKNMiJ [6], Allen [7], Rondal and Maquoi [8]).

For logarithmic-normal distribution parameters: $\breve{\varphi}, v_{\varphi}$ and design value $\varphi_{d}$ of buckling coefficient $\underline{\varphi}$ are related as follows

$$
\begin{aligned}
\varphi_{d} \stackrel{d f}{=} N_{b . R d} / N_{p l, d} & =\breve{N}_{b . R} \cdot \exp \left(-\beta_{R} \cdot \sqrt{v_{\varphi}^{2}+v_{R}^{2}}\right) /\left(\breve{N}_{p l} \cdot \exp \left(-\beta_{R} \cdot v_{R}\right)\right)= \\
& =\breve{\varphi} \cdot \exp \left(\beta_{R} \cdot\left(v_{R}-\sqrt{v_{\varphi}^{2}+v_{R}^{2}}\right)\right) .
\end{aligned}
$$

Introducing into Eq. 3 values according formula (2) and Table 2, $v_{R}=0,10$ (according to (Murzewski [9])) for random plastic resistance of steel $\underline{R} \equiv \underline{f} \quad\left(\underline{R}=\underline{N}_{p l} / \underline{A}\right)$ corrected for random deviation of bar cross-section area $A$ and partial reliability index $\beta_{R}=3,0$ relationship $\varphi_{d}(\Lambda)$ was received. This relationship was compared (for buckling curve "b") in Figure 3 with other curves characterizing buckling coefficient.

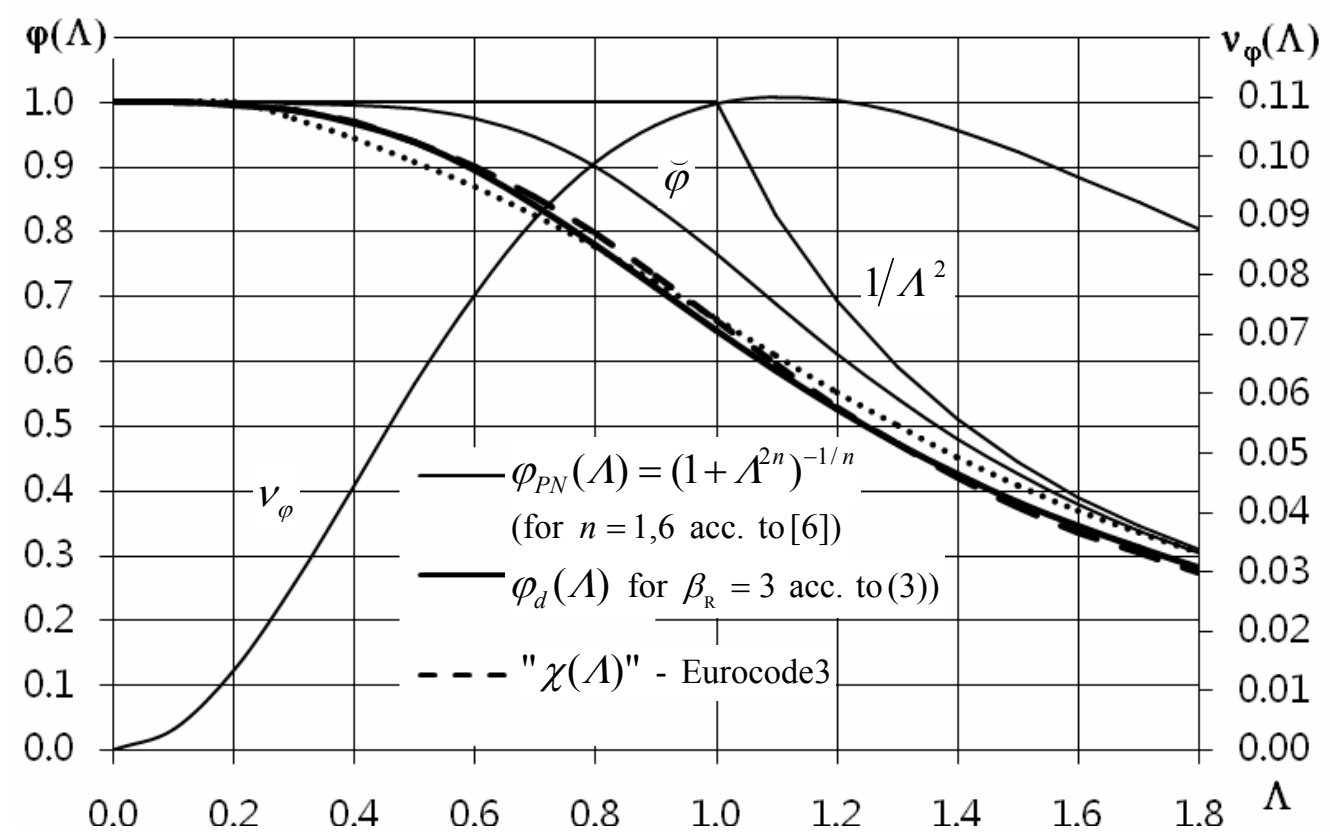

Figure 3. Parameters $\breve{\varphi}$ and $v_{\varphi}$ and Different Relationships $\varphi_{d}(\Lambda)$ for Buckling Curve "b"

Design values of $\varphi_{d}$ obtained from Eq. 3 for $\beta_{R}=3,0$ and parameters according to Table 2 are very close to standard values of $\varphi_{P N}(\Lambda)$ from Polish Standard [6] and values of $\chi(\Lambda)$ from Eurocode [5] (it was shown in Figure 3 - for buckling curve "b") if following relationship will be used

$$
\Lambda=1,1178 \cdot \bar{\lambda} \cong 1,12 \cdot \bar{\lambda}
$$

Relationship (4) may be obtained by assuming in general formula for relative slenderness

$$
\lambda_{r} \stackrel{d f}{=} \sqrt{N_{p l} / N_{E}} \cong \lambda /\left(\pi \cdot \sqrt{E / R_{p l}}\right)=\lambda / \lambda_{1}
$$


medians: $E=\breve{E}=205 \mathrm{GPa}$ and $R_{p l}=\breve{f}=290 \mathrm{MPa}$ (hence $\lambda_{1}^{P N}=84$ ) - as it was made in Polish Standard [6] for low-carbon steel or values: $E=210 \mathrm{GPa}$ and $R_{p l}=f_{y}=235 \mathrm{MPa}$ (hence $\lambda_{1}^{E C}=93,9$ ) - according to Eurocode [5].

To determinate column random equivalent initial bow we use well-known formula of dimensionless equivalent initial deflection $\varepsilon_{0}$ (Rondal And Maquoi [8], ECCS [10]) of hinged bar with sinusoidal initial bow (the formula is based on bar resistance conservation criterion)

$\varepsilon_{0 e}=e_{0 e} / r=\left(\varphi^{-1}-1\right) \cdot\left(1-\varphi \cdot \Lambda^{2}\right)=\varphi^{-1}+\varphi \cdot \Lambda^{2}-\left(1+\Lambda^{2}\right)$

where: $e_{0 e}$ - bar equivalent initial bow,

$r \equiv W / A \quad$ - radius of cross-section core.

Random imperfection $\varepsilon_{0 e}=\underline{e}_{0 e} / r$ is obtained utilizing randomization of Eq. 6 by introducing in place of $\varphi$ random bucking coefficient $\underline{\varphi}$ with logarithmic-normal distribution and parameters according to formulas (2) and Table 2 . It follows that we have to determinate $\underline{\varepsilon}_{0 e}$ probability distribution as a known deterministic function $\varepsilon_{0 e}(\underline{\varphi})$ of the known random variable $\underline{\varphi}$.

According to mechanical interpretation and property of logarithmic-normal distribution quantity $\varepsilon_{0 e}=e_{0 e} / r$, similarly as other quantities in Eq. 6, are treated as non-negative (or positive) what follows that function $\varepsilon_{0 e}(\varphi)$ is determined for $\varphi \in\left(0, \min \left(1,1 / \Lambda^{2}\right)\right]$. In this range of $\varphi$ values function $\varepsilon_{0 e}(\varphi)$ is monotonically decreasing (Figure 4(a)) and probability density function $g\left(\varepsilon_{0 e}\right)$ amounts to transformation type (Papoulis [11]):

$g(y)=f\left(u^{-1}(y)\right) \cdot\left|\frac{d u^{-1}(y)}{d y}\right|$

where: $f(x)$ - known probability density function of random variable $\underline{x}$,

$u^{-1}\left(y_{x}\right)$ - inverse function of given function $y=u(x)$ - strictly monotonic,

$g(y)$ - inquired probability density function of random variable $\underline{y}=u(\underline{x})$.

Logarithmic-normal distribution, rational in case of coefficient $\varphi$ (definite for $\varphi>0$ and stable in relation to multiplication) does not fulfil yet condition $\varphi \leq \varphi_{\max }=\min \left(1,1 / \Lambda^{2}\right)$. To calculate density function $g\left(\varepsilon_{0 e}\right)$ we introduce corrected (cut off) distribution $\underline{\varphi}$ with density $c \cdot f(\varphi)$, where: $c=1 /\left(1-\omega_{m}\right)$ - normalizing factor, $\omega_{m}=$ Prob. $\left\{\varphi \geq \varphi_{\max }\right\}$ (Figure 4(a)). This adoption we propose for skeleton column slenderness $\Lambda \leq \sim 1,4$ (that is $\lambda<\sim 120$ ). For slenderness values distant from 1 coefficient $c$ increases significantly.

After introducing into Eq. 7 function $\varepsilon_{0 e}(\varphi ; \Lambda)$ and corrected logarithmic-normal probability density function $f(\varphi ; \Lambda)$ with parameters $\varphi(\Lambda)$ and $v_{\varphi}(\Lambda)$ we obtain density function of random variable $\underline{\varepsilon}_{0 e}$ in the form of: 
$g\left(\varepsilon_{0 e} ; \Lambda\right)=\frac{c}{\sqrt{2 \pi} \cdot v_{\varphi} \cdot \varphi\left(\varepsilon_{0 e}\right)} \cdot \exp \left\{-\frac{\ln ^{2}\left(\varphi\left(\varepsilon_{0 e}\right) / \breve{\varphi}\right)}{2 \cdot v_{\varphi}^{2}}\right\} \cdot\left|\frac{\mathrm{d} \varphi\left(\varepsilon_{0 e}\right)}{\mathrm{d} \varepsilon_{0 e}}\right|$,

where: $\varphi\left(\varepsilon_{0 e}\right)=(a-b) /\left(2 \cdot \Lambda^{2}\right), \mathrm{d} \varphi / \mathrm{d} \varepsilon_{0 e}=(1-a / b) /\left(2 \cdot \Lambda^{2}\right), \quad a=a\left(\varepsilon_{0 e} ; \Lambda\right)=\varepsilon_{0 e}+\Lambda^{2}+1$,

$$
b=b\left(\varepsilon_{0 e} ; \Lambda\right)=\varepsilon_{0 e}^{2}+2 \cdot \varepsilon_{0 e} \cdot\left(1+\Lambda^{2}\right)+\left(1-\Lambda^{2}\right)^{2} .
$$

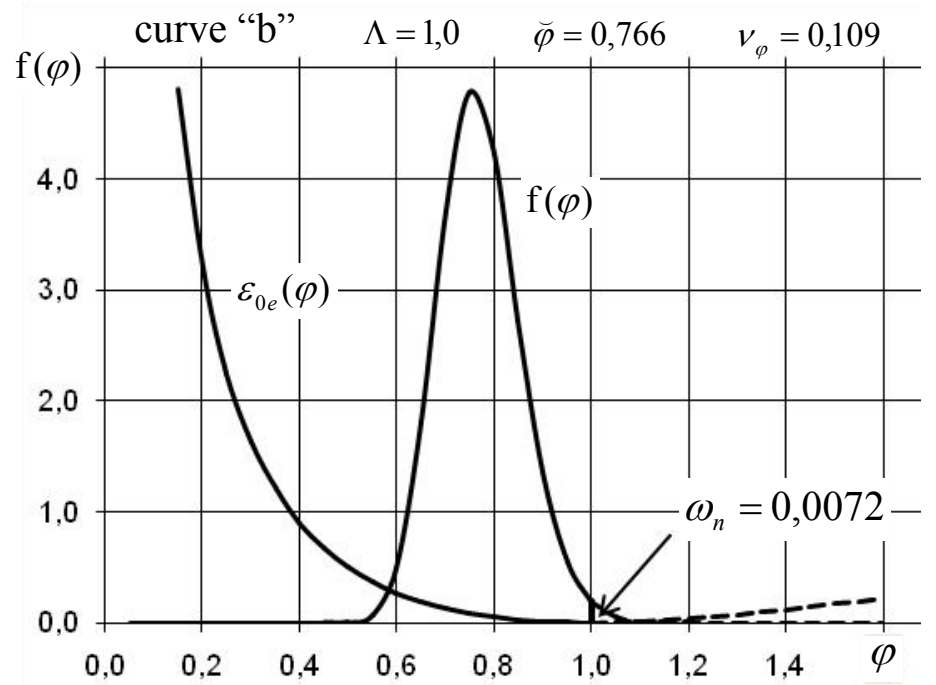

Figure 4(a). The Example of $\varepsilon_{0 e}(\varphi)$ and $f(\varphi)$ Relationship

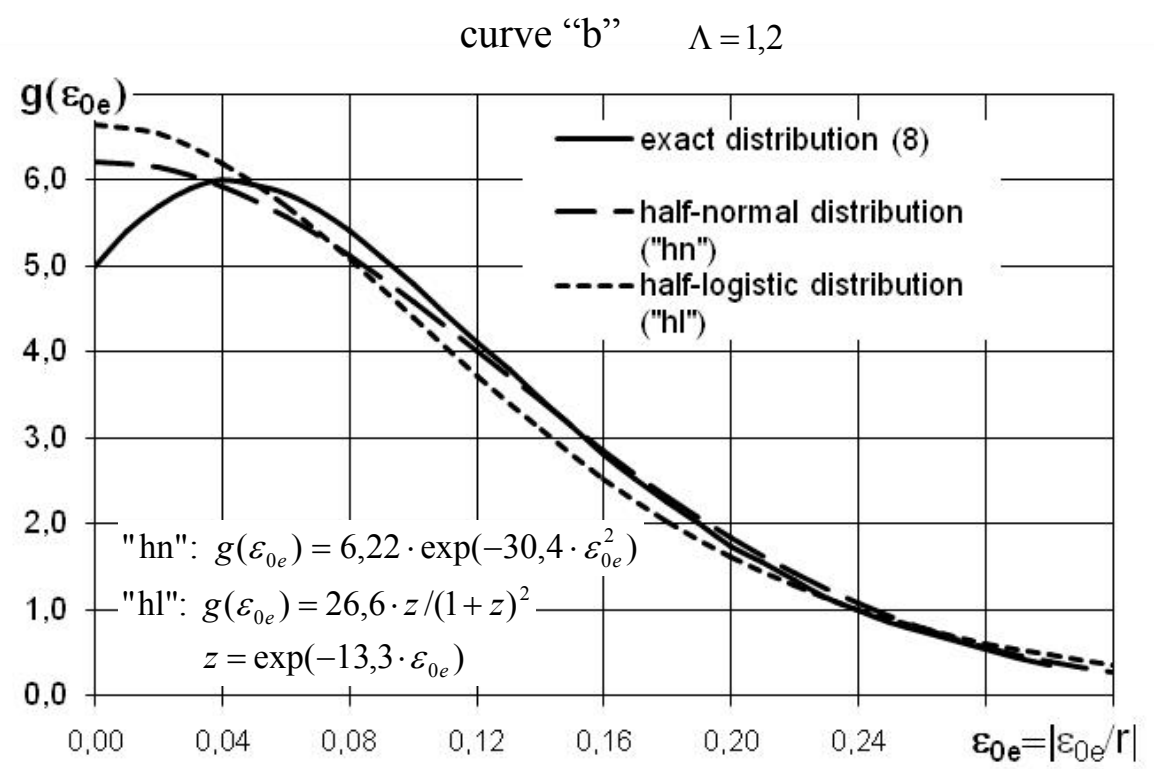

Figure 4(b). The Example of Probability Density Function $g\left(\varepsilon_{0 e}\right)$

Example results of calculation according to Eq. 8, for bucking curve "b" and $\Lambda=1,2$, were shown in Figure 4(b). Density of "exact" distribution ("8") according to Eq. 8 shows (beside of close surroundings of $\varepsilon_{0 e}=0$ point) very good compatibility with density of half-normal distribution ("hn") and good compatibility with density of half-logistic distribution ("hl"). One-parameter half distributions mentioned above were matched according to mean values conformity criterion: $\bar{X}_{e}=\bar{X}_{h n}=\bar{X}_{h l} \quad\left(\right.$ where $\left.\bar{X} \equiv \bar{\varepsilon}_{0 e}\right)$. 
Mean values $\bar{\varepsilon}_{0 e}$ and standard deviations $\mu_{\varepsilon 0 e}$, determined from numerical integration of Eq. 8, for appropriate half-normal distributions $\underline{\varepsilon}_{0 e}$, are related to $\Lambda$ almost linearly (Figure 5(a))

$\bar{\varepsilon}_{0 e}=C_{\bar{\varepsilon}} \cdot \Lambda, \quad \mu_{\varepsilon 0 e}=C_{\mu \varepsilon} \cdot \Lambda$,

with direction coefficients $C_{\bar{\varepsilon}}$ and $C_{\mu \varepsilon}$ given in Table 3.

Value of coefficient $C_{\mu \varepsilon}^{*}$ (for relationship $\mu_{\varepsilon 0 e}^{*}=C_{\mu \varepsilon}^{*} \cdot \Lambda$ ) corresponding to normal distribution $\underline{\varepsilon}_{0 e}^{*}$ with changeable signs and mean value $\bar{\varepsilon}_{0 e}^{*}=0$ is obtained (Table 3) from relationship $C_{\mu \varepsilon}^{*}=C_{\mu \varepsilon} / \sqrt{1-2 / \pi}$ (Machowski [3]).

In Figure 5(b) values of $\varepsilon_{0 e}(\Lambda)$, obtained from half-normal distribution $\varepsilon_{0 e}$ with parameters according to Table 3, were compared, for bucking curve "b", with values from Standard Recommendations and values from formula (6). Straight lines "1" and "2" are obtained for assumed probability $\omega$ of exceeding $\varepsilon_{0 e}$ value (top quantiles) in half-normal distribution. Straight line " 2 " corresponding to value $\omega=2 \%$ turns out very close to straight line " 3 " characterized by equation $\varepsilon_{0 z}=0,3 \cdot \lambda / 100=0,3 \cdot \Lambda \cdot \lambda_{1} / 100$ (for $\lambda_{1}=0,84$ ) - representing traditional British Recommendations (BS 449). Broken line " 4 ", according to Eurocode 3 - with equation: $\varepsilon_{0 z}=0,34 \cdot(\bar{\lambda}-0,2) \quad$ - for $\bar{\lambda}>0,2$ and $\varepsilon_{0 z}=0 \quad$ - for $\bar{\lambda} \leq 0,2$, (taking relationship $\Lambda=1,12 \cdot \bar{\lambda}$ into consideration) shows good compatibility with curve "5" obtained from (6) substituting $\chi(\bar{\lambda})$ in place of $\varphi(\lambda)$ and $\bar{\lambda}$ in place of $\Lambda$.

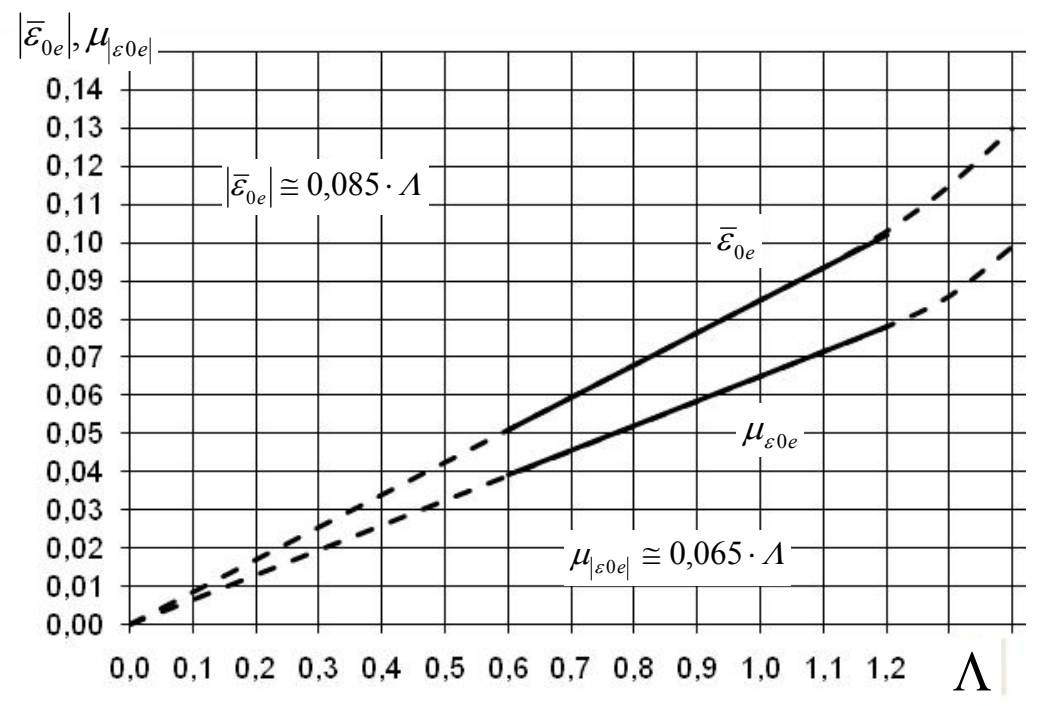

Figure 5(a). Examples of Relation $\bar{\varepsilon}_{0 e}(\Lambda)$ and $\mu_{\varepsilon 0 e}(\Lambda)$ for Curve "b" 


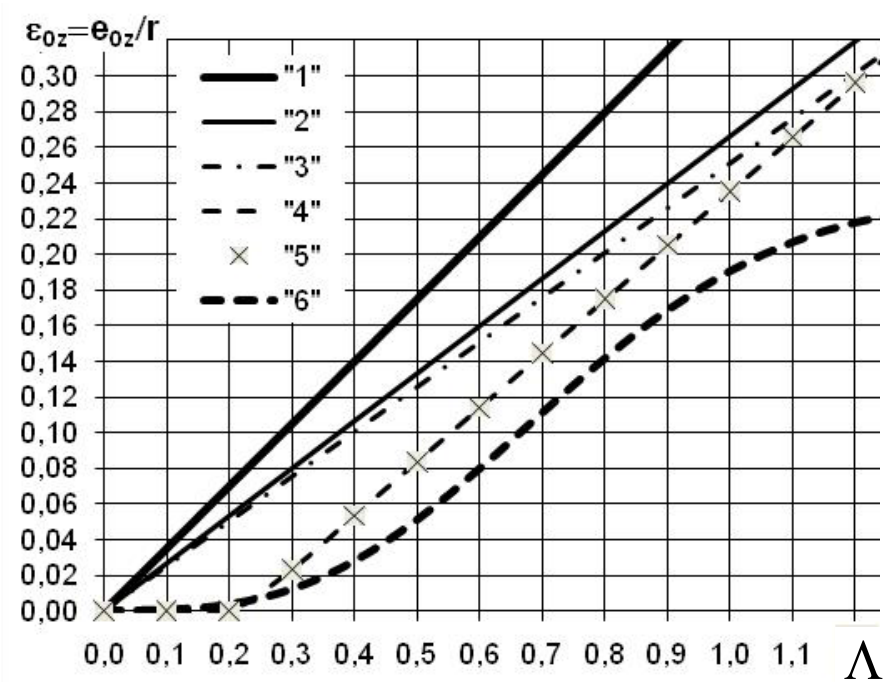

Figure 5(b). Comparison of relation $\varepsilon_{0 e}(\Lambda)$ according to different formulas

("1" - random for $\omega=1,35 \%$, " 2 " - random for $\omega=2 \%, " 3 "-$ " $0,3 \lambda / 100 "$, " $4 "$ - " $0,34(\bar{\lambda}-0,2) "$, " $5 "-$ acc. to Eq. 6 for $\bar{\lambda}$ and $\chi(\bar{\lambda}), " 6 "-$ acc. to Eq. 6 for $\Lambda$ and $\varphi(\Lambda)$

Table 3. Coefficients values for formula (9)

\begin{tabular}{|l|l|l|l|}
\hline $\begin{array}{l}\text { buckling } \\
\text { curve }\end{array}$ & $C_{\bar{\varepsilon}}$ & $C_{\mu \varepsilon}$ & $C_{\mu \varepsilon}^{*}$ \\
\hline $\mathrm{a}$ & 0,065 & 0,045 & 0,075 \\
\hline $\mathrm{b}$ & 0,085 & 0,065 & 0,110 \\
\hline $\mathrm{c}$ & 0,120 & 0,085 & 0,140 \\
\hline
\end{tabular}

Occurrence of $\varphi$ and $\Lambda$ quantities in non-linear form in Eq. (6) cause significant mutual discrepancy between curves " 5 " and "6" (Figure 5(b)) despite of affirmed earlier good compatibility of buckling curves (Figure 3).

\section{RANDOM EQUIVALENT INITIAL TILT OF FRAME}

Mathematical model of column single story initial tilt was determined on the basis of column out-of-plumbs post-fabrication measurements, carried out after all frames erection and after construction of all floors.

Most extensive statistical analysis of measurements mentioned above was conducted in (Machowski $[3,12]$ ) which also includes results obtained earlier by Beaulieu (Beaulieu and Adams [13]) and Lindner (Lindner and Gietzelt [14]).

Conducted analysis showed that that mathematical model of stationary gaussian random noise $\underline{\phi}_{i j k}^{x}$ and $\underline{\phi}_{i j k}^{y}$ (stationary random series with mean value $\bar{\phi}=0$ and constant variance $D_{\phi}=\mu_{\phi}^{2}=$ const. ) is justified if empirical variance fulfils a constraint

$\mu_{\phi}^{2} \leq 3(\%)^{2}$,

what agrees with keeping initial tilt standard tolerance. 
The second conclusion was that only exist weak autocorrelation dependence between tilts of columns in the same vertical planar frames and lack of correlation between any other initial tilts.

For design purposes it is very convenient to assume one random value of equivalent initial tilt for frame as a whole $\underline{\phi}^{E F F}$ instead of column initial tilts random sequences $\underline{\phi}_{i p}$.

Post-fabrication column out-of-plumbs cause additional bending moments from vertical load for frame as vertical cantilever (Machowski, A. [3, 12]).

For bar-disk analytical scheme of multistory skeletal structure with rigid floor-disks effective initial random tilt for $i$-th story of building $\phi_{i}^{\text {eff }}$ may follow from conservation of global moment increment condition (if torsional effect for building as vertical cantilever is neglected)

$$
\Delta \underline{\mathrm{M}}_{\mathrm{i}}^{\phi}=\sum_{\mathrm{p}=1}^{\mathrm{s}} \mathrm{P}_{\mathrm{ip}} \cdot \mathrm{h}_{\mathrm{i}} \cdot \underline{\phi}_{\mathrm{ip}}=\mathrm{P}_{\mathrm{i}} \cdot \mathrm{h}_{\mathrm{i}} \cdot \underline{\phi}_{\mathrm{i}}^{\mathrm{eff}}
$$

where: $P_{i}=\Sigma \mathrm{P}_{i p},(p=1 \ldots s$.$) - sum of all vertical loads acting on building above i$-th story, $P_{i p}($ for $s$ columns $i=1,2, \ldots, s$. on $i$-th story of building) - sum of vertical loads above $i$-th story in $p$-th column,

hence $i$-th story equivalent initial tilt

$$
\underline{\phi}_{i}^{e f f}=\sum_{p=1}^{s} w_{i p} \cdot \underline{\phi}_{i p}=\mathbf{w}_{i} \boldsymbol{\phi}_{i}^{\mathrm{T}}
$$

Story random initial tilt $\underline{\phi}_{i}^{\text {eff }}$ is normal random variable with parameters (Machowski [3, 12]):

$$
\mathrm{E}\left\{\phi_{i}^{e f f}\right\}=0, \quad \mu_{\phi \mathrm{i}}^{2}=k_{c, i}^{2} \cdot \mu_{\phi}^{2}
$$

where

$$
k_{c, i}^{2}=\mathbf{w}_{i} \mathbf{\rho}_{s} \mathbf{w}_{i}^{\mathrm{T}}=\sum_{p=1}^{s} w_{i p} \cdot \sum_{q=1}^{s} w_{i q} \cdot \rho_{p q}
$$

is positively defined quadratic form of $s$-variables: $\mathrm{W}_{\mathrm{i} 1}, \ldots, \mathrm{W}_{\mathrm{ip}}, \ldots \mathrm{W}_{\mathrm{is}}$. with symmetric matrix of coefficients $\rho_{\mathrm{s}}$ equal to normalized correlation matrix $\rho_{\mathrm{s}}$ of random vector $\phi_{\mathrm{i}}$.

Values of reduction coefficient $k_{c, i}$ according to Eq. 14 have been analysed in detail in (Machowski $[3,12])$. Conducted analysis showed that among standard formulas closest to obtained result is that in Polish Standard [6], on condition that only columns with vertical load (above $i$-th story) arrangement parameter $c \geq 0,5$ are taken into account.

Safe approximation of effective random initial tilt for frame as a whole may be defined as follows 


$$
\underline{\phi}^{E F F}=\max _{i=1}^{n}\left|\underline{\phi}_{i}^{E F F}\right|=\max _{i=1}^{n}\left|\sum_{t=i}^{n} V_{t} \cdot \sum_{q=i}^{t} h_{q} \cdot \underline{\phi}_{q}^{e f f} \cdot\left(\sum_{t=i}^{n} V_{t} \cdot \sum_{q=i}^{t} h_{q}\right)^{-1}\right|,
$$

$\underline{\phi}_{i}^{E F F}$ - effective random initial tilt for frame as a whole, which guarantee safe estimation of additional bending moment for $i$-th story of frame as vertical cantilever,

$\underline{\phi}_{i}^{e f f}$ - according to Eq. 12 ,

$V_{t}$ - sum of vertical loads imposed immediately to $t$-th story.

Analyses conducted in (Machowski [3, 12]) showed that within confines of discussed conception reduction of initial tilt standard deviation and characteristic value depending on story number is unfounded. According to extensive analyses presented in (Rondal and Maquoi [8], Machowski and Tylek [15]) this reduction is justified only in special cases, e.g. with reference to frames fulfilled "strong columns" condition (columns remain elastic up to plastic mechanism formation).

\section{JOINT EFFECT OF EQUIVALENT INITIAL TILT AND INITIAL BOW}

Statistically based models of equivalent imperfections $\underline{e}_{0 e}$ and $\underline{\phi}_{0 e}$, according to chapters 2 and 3 , first of all may be used to verify Standard conceptions of steel skeletons calculation. This verification may apply, among others things, to often criticized effective length conception for sway frames and described in p. 6.3.4 Eurocode 3 [5] so called "general method" depending on reduction of resistance coefficient $\alpha_{u l t, k}$ by means of factor $\chi_{o p}$ determined as Standard flexural buckling coefficient $\chi$ or Standard lateral-torsional buckling coefficient $\chi_{L T}$ - calculated for "global non-dimensional slenderness" $\bar{\lambda}_{o p}$, connected with out-of-plane buckling of a structural component. Analysis of Standard conceptions mentioned above with application of proposed models of $\underline{e}_{0 e}$ and $\underline{\phi}_{0 e}$ would require, as it seems, separate extensive study.

Another important problem that will be consider farther in this article, is influence of both random equivalent imperfections on total imperfections effect. In this case random variability of $\underline{e}_{0 e}$ sign (direction) in column assembly elements may be important. Taking into account of this last effect requires, even in the simplest cases, to consider examples of multistory steel frames.

Joint effect of random equivalent initial tilt $\underline{\phi}_{0 e}$ and random equivalent initial bow $\underline{e}_{0 e}$ in "advanced analysis" was considered on the example of 5-story steel skeleton (Figure 6). Vertical loads from floors, roof and walls of the building are carried by floor beams on five transversal rigid frames located in axes 1-1 to 5-5 of building horizontal projection. Skeleton bracing system, carrying horizontal load, is made up, among transversal frames mentioned above, three equal two-bay rigid frames located in axes: A-A, B-B and C-C (see horizontal projection in Figure 6). Skeleton filling consist of floors and flat roof with reinforced concrete supporting structure, (rigid in own plane and nonflexibly connected with beams) and light-weight partition and curtain walls. It should be noticed that plane of every longitudinal frame agrees with column minor stiffness plane (Figures 6 and 7). 


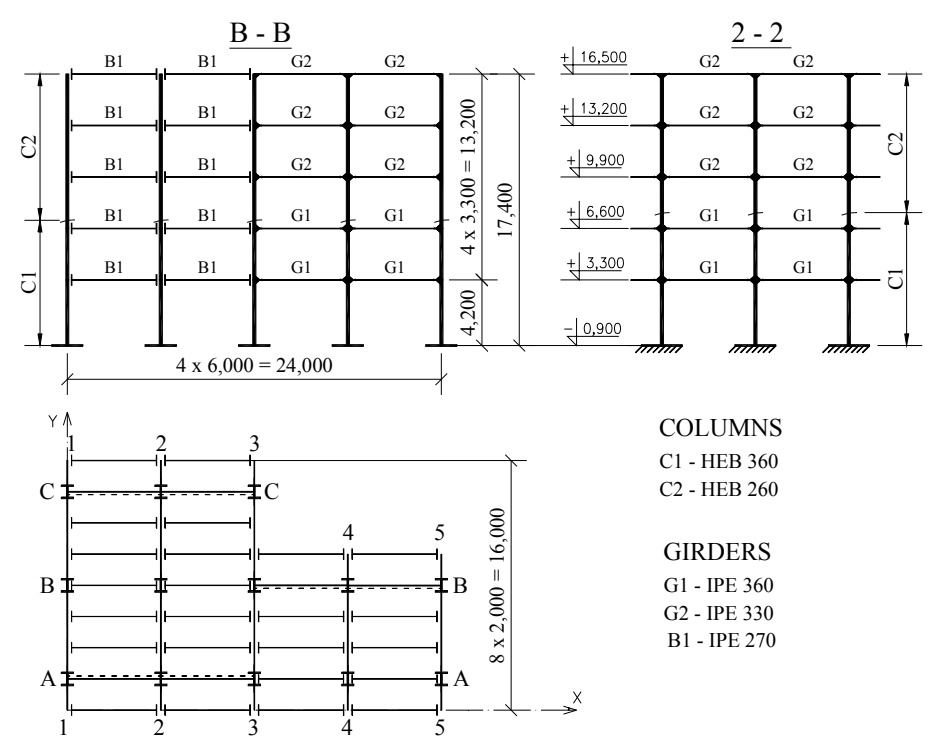

Figure 6. Analysed Skeletal Framed Steel Structure

Considering vertical planar frame of longitudinal bracing system (e.g. C-C) acc. to Figure 6 and neglecting torsional effects for building as vertical cantilever substitute static system of planar frame (Figure 7) was assumed. This system was loaded by realistic vertical loads in the form of concentrated forces $Q_{11}^{C}$ to $Q_{n 3}^{C}$ - putted in nodes and fictitious imperfectional load uniformly distributed on columns: $q_{11} \ldots q_{n 3}$ (as in [5]) - balanced by reactions $R_{11} \ldots R_{n 3}$.

Influence of column initial tilts of skeleton braced in longitudinal direction by three identical frames, joined by rigid floor disks, was taken into consideration by adding to planar frame fictitious system (Figure 7) consisted of bars ideally rigid, connected by hinges with each other and with frame. To the nodes of system mentioned above following loads were applied: vertical loads $V_{1} \ldots$ $V_{i} \ldots V_{n}$ equal to $1 / 3$ of sum of $Q_{i}$ vertical loads on $i$-th skeleton floor and couples of forces $P_{i} \phi_{0 i}(i$ $=1,2, \ldots, n$.) representing equivalent imperfectional load from initial tilts.

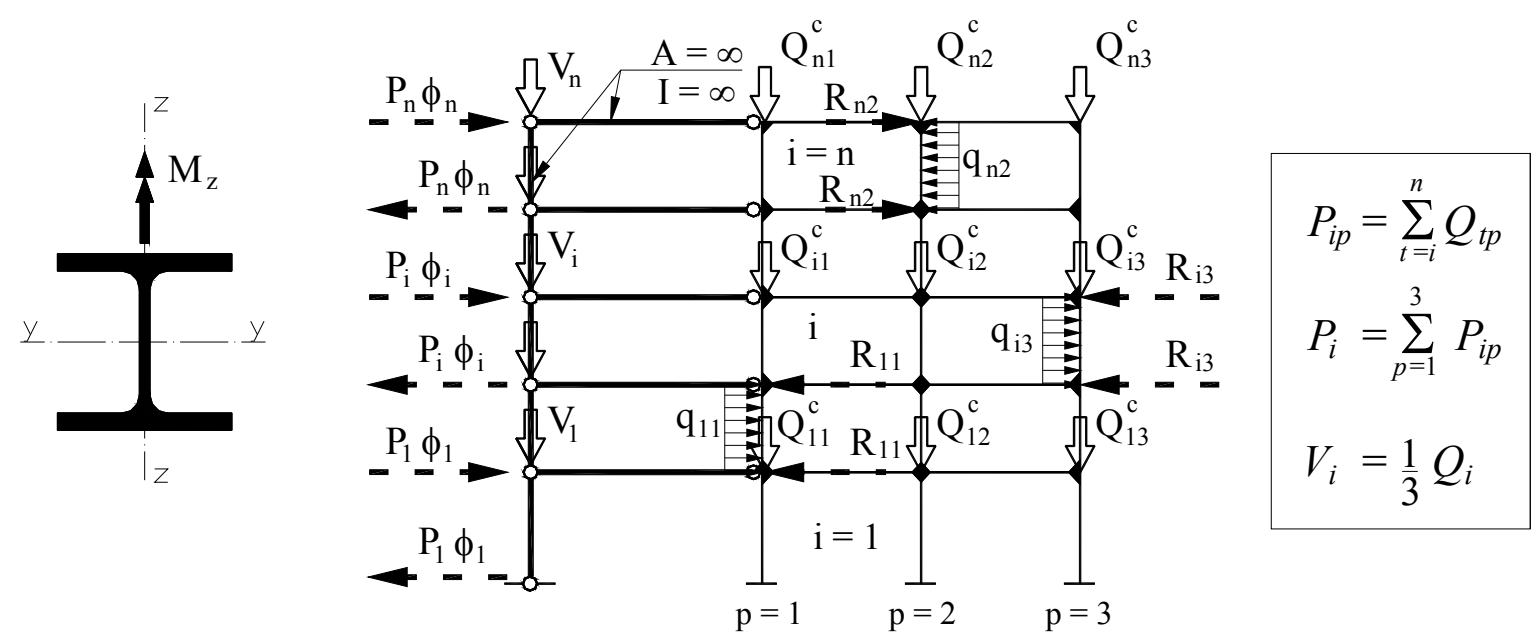

Figure 7. Substitute Static System of Analyzed Planar Bracing System of 5-story Skeletal Steel Structure $\left(Q_{i p}-\right.$ Total Vertical Load of Building as a Whole $i$-th story) 
Random sequences of story initial tilts were computer generated in the form of vector $\underline{\phi}^{\text {eff }}=\left\{\underline{\phi}_{i}^{\text {eff }}\right\}$ which elements were normal variable with parameters according to Eq. 13. Week correlation between initial tilts in vertical sequences was neglected.

Mathematical model of column random equivalent initial bow was product of random initial bow and random binary sequence (with elements 1 and -1). Value of column random initial bow was determined on the basis of knowledge of probability density function and characteristics of random dimensionless column bow distribution (according to p.2). Normal distribution with parameters $\left\{\bar{\varepsilon}_{0 e}^{*}=0, \mu_{\varepsilon 0 e}^{*}=C_{\mu \varepsilon}^{*} \cdot \Lambda\right\} \quad\left(C_{\mu \varepsilon}^{*}\right.$ according to Table 3$)$ was assumed. It was also assumed that column splices are located on every story (above bottom node of column) so there is no correlation between initial bows of individual columns.

Additional effect of column equivalent initial bows on frame carrying capacity (associated with limit point on frame equilibrium path) was determined by comparison of frame initial tilt $\underline{\phi}^{E F F}\left(\underline{\phi}_{0 e}\right)$ (substitutes for random sequence of story initial tilts only - Figure 8a) standard deviation with frame initial tilt $\underline{\phi}^{E F F}\left(\underline{\phi}_{0 e}, \underline{e}_{0 e}\right)$ (substitutes for story random initial tilts and column random initial bows - Figure $8 b$ ) standard deviation values. In both cases the same sequences of story random initial tilts were considered.

a)

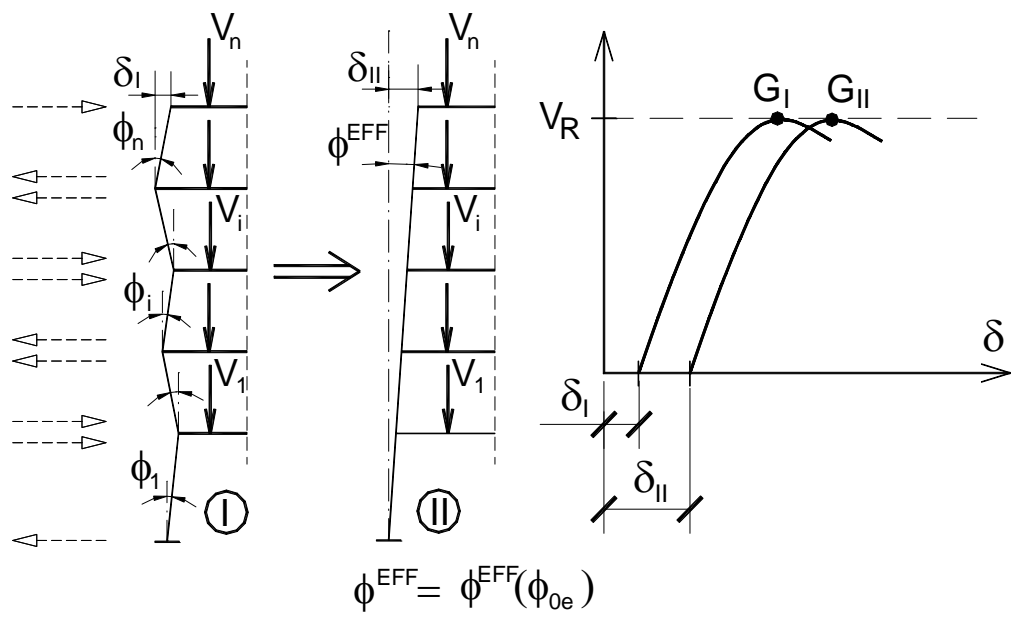

b)
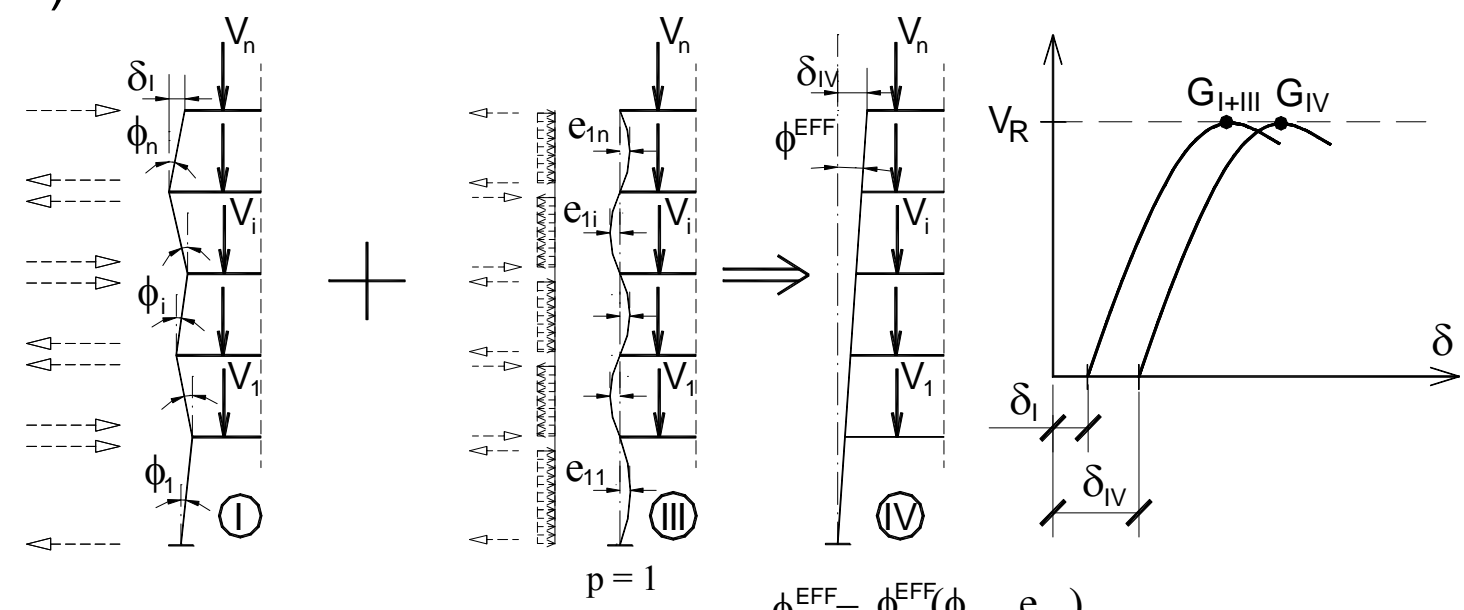

$\phi^{\mathrm{EFF}}=\phi^{\mathrm{EFF}}\left(\phi_{0 \mathrm{e}}, \mathrm{e}_{0 \mathrm{e}}\right)$

Figure 8. Random Sequence of Story Initial Tilts, Random Set of Column $p=1$ Initial Bows and Its Equivalent Forces; Equivalent Tilts of Frame as a Whole and Corresponding Paths of Equilibrium 
According to applied simulation procedure, for every realization of equivalent imperfectional loads set (Figure 7) respective frame equilibrium path and frame carrying capacity were determined. Next the effective initial tilt of frame as a whole $\phi^{E F F}$ was chosen in agreement with condition of frame limit carrying capacity conservation (Figure 8). Equivalent random initial tilt of plane frame as a whole $\phi^{E F F}$ was determined on the basis of conservation of carrying capacity associated with limit point on frame equilibrium path criterion. Calculations were conducted for 700 elements population.

In order to obtain frame equilibrium path and frame limit carrying capacities computer program ANSYS, based Finite Element Method, were utilized. Two-dimensional bar structure model with regular mesh and elastic-plastic (bilinear) model of material was assumed. Description of the material model was based on Huber-Mises-Hencky plasticity criterion, associative flow rule and rule of kinematic hardening. Development of plastic zones (in cross section and along length of element) and large displacements (translations and rotations) with small deflections were take into consideration.

Difference between standard deviations of frame initial tilt $\underline{\phi}^{E F F}\left(\underline{\phi}_{0 e}\right)$ and frame initial tilt $\underline{\phi}^{E F F}\left(\underline{\phi}_{0 e}, \underline{e}_{0 e}\right)$ obtained from conducted analysis is lower than $2 \%$ and indicates that additional influence of column random equivalent initial bows on frame carrying capacity in advanced analysis may be neglected.

Results of earlier works (De Luca and Mele [17]) indicated that interaction of global (tilts) and local (bows) geometric imperfections causes reduction of frame carrying capacity by $10 \%$, but authors [17] assumed deterministic (instead of random) values of equivalent imperfections and selected some combinations of global and local equivalent geometric imperfections, compatible with first buckling eigenvector of ideal elastic frame and combinations of initial bows acceptable for the sake of columns splices (Figure 9).

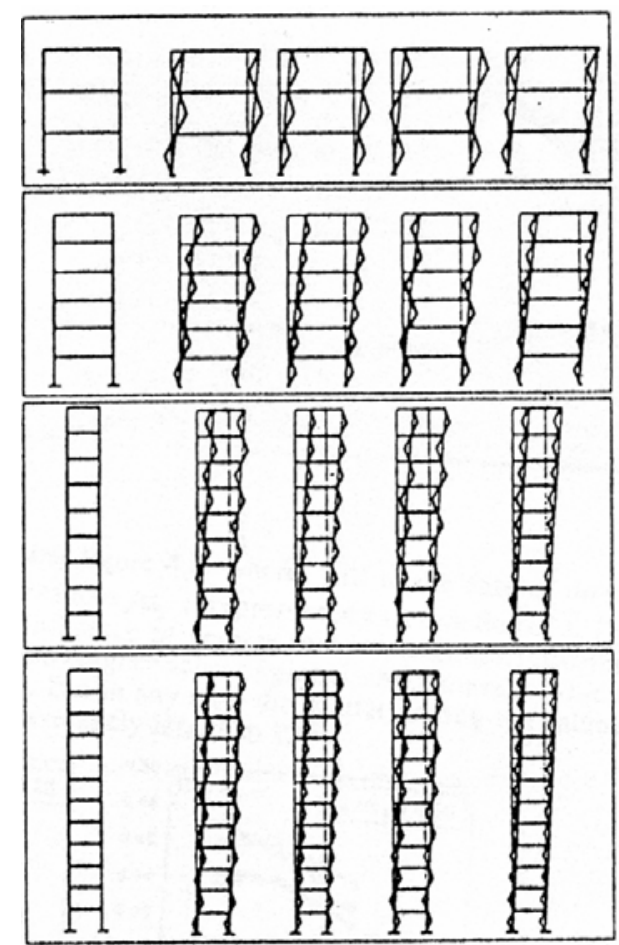

Figure 9. Set of Local and Global Geometrical Imperfections Combination Analyzed in [17] 


\section{CONCLUSIONS}

Verification of Standard recommendations of steel skeletons design with equivalent geometrical imperfections (column initial tilt $\phi_{0}$ and bow $e_{0}$ ) should base on probabilistic models of these imperfections and takes into account its interaction. Application of random models and imperfections acting together should lead to taking into consideration of favourable effects similar to these which we deal with in case of random load combinations.

Possibility of applying approach mentioned above offers this work model of equivalent random initial bow of compressed bar $\underline{e}_{0}$. It is obtained out of randomization of buckling coefficient in well-known deterministic formula of equivalent initial bow. This model together with known from earlier works, e.g. authors' works $[3,12,15,16]$, model of equivalent random initial tilt $\underline{\phi}_{0}$, allows analyses of mentioned above Standard reccomendations.

In the paper effects of column initial tilts and initial bows interaction were investigated using probabilistic approach, introducing into calculations, in elastic-plastic structure behaviour, statistically based models of equivalent imperfections.

Analysis of 2-bay 5-story frame planar bracing system with random sequences of story initial tilts and random sets of column initial bows showed slight influence of column random initial bows on frame carrying capacity and equivalent initial tilt of frame as a whole $\underline{\phi}^{E F F}$. Influence of interaction of global and local imperfections on frame carrying capacity was lower than $2 \%$.

Results of conducted analysis justify passing over of local geometric imperfections influence in planar frames advanced analysis. It also shows that Eurocode 3 procedure of steel frame design, recommending to introduce in stage on static calculations only frame equivalent initial tilt while influence of column equivalent initial bows is taking into account in beam-column design formulas.

\section{ACKNOWLEDGEMENT}

Scientific research has been carried out as a part of the Project "Innovative resources and effective methods of safety improvement and durability of buildings and transport infrastructure in the sustainable development" financed by European Union from the European Fund of Regional Development based on the Operational Program of the Innovative Economy.

\section{REFERENCES}

[1] Chen, W.F. and Toma, S., “Advanced Analysis of Steel Frames”, London-Tokyo: CRC Press, 1994.

[2] Narayanan, R. ed, "Steel Framed Structures, Stability and Strength", Elsevier Science, 1985.

[3] Machowski, A., "Problems of Limit States and Reliability of Steel Multistory Building Frames", Monograph No. 262, Cracow University of Technology, 1999 [in Polish].

[4] Fukumoto, Y. and Itoh, Y., "Multiple Buckling Curves Based on Experimental Data", Construction Metállique, 1984, Vol. 3 [in French].

[5] CEN "EN 1993-1-1. Eurocode 3, Design of Steel Structures, Part 1.1: General Rules and Rules for Buildings".

[6] PKNMiJ, “PN-90/B-03200, Steel Structures, Design Rules” [in Polish]. 
[7] Allen, D., "Merchant-Rankine Approach to Member Stability", Journal of the Structural Division, 1978, 104 (ST.12), pp. 1909-1914.

[8] Rondal, J. and Maquoi, R., "The Ayrton-Perry Formulation for Buckling of Metallic Bars", Construction Metállique, 1979, 4, pp. 41-53 [in French].

[9] Murzewski, J., "Design of Steel Structures for Differentiated Reliability Levels", Archives of Civil Engineering, 2008, LIV(1), pp. 209-237.

[10] ECCS, "European Specifications for Steel Structures", 1-st ed; 1974.

[11] Papoulis, A., "Probability, Random Variables and Stochastic Processes", McGraw-Hill Inc, 1965.

[12] Machowski, A., "Initial Random Out-of-plumbs of Steel Frame Columns", Archives of Civil Engineering, 2002, XLVIII(2), pp. 207-226.

[13] Beaulieu, D. and Adams, P.F., "The Results of a Survey on Structural Out-of-plumbs", Canadian Journal of Civil Engineering, 1978, Vol. 5, pp. 642-470.

[14] Lindner, J. and Gietzelt, R., "Evaluation of Imperfections of Support - Elements", Stahlbau, 1984, Vol. 4, pp. 97-98 [in German].

[15] Machowski, A. and Tylek, I., "Conceptions of Equivalent Imperfections in Analysis of Steel Frames", The International Journal of Advanced Steel Construction, 2008, Vol. 4, No. 1, pp. $13-25$.

[16] Tylek, I., "Equivalent Geometrical Imperfections of Multistory Steel Building Frames", Doctoral Thesis, Cracow University of Technology, 2007 [in Polish].

[17] De Luca A. and Mele, E., "Analysis of Steel Frames in the Light of Eurocode 3 and New Research Results", In: White, D.W. and Chen, W.F. ed., "Plastic Hinge Based Methods for Advanced Analysis and Design of Steel Frames", SSRC, Lehigh Univ., 1993, pp. 97-152. 